\title{
Reanimación cardiopulmonar presenciada en el contexto hospitalario mexicano: un estudio de caso en pacientes y familiares
}

\author{
Javier Eduardo García de Alba García, Juan Francisco García \\ Regalado y Ana L. Salcedo Rocha
}

En este artículo se plantea el estudio de la reanimación cardiopulmonar presenciada (RCPP) como objeto de análisis en el marco de las reconfiguraciones culturales actuales. De la opinión de 90 pacientes que ingresaron a Urgencias y 90 familiares que aceptaron participar en una encuesta, se encontró consenso cultural entre los familiares y pacientes a favor de la RCPp. Se rescata esta experiencia a partir de una mirada que va más allá de la racionalidad técnica, para establecer algunas bases que puedan dar origen a una política humanística que reconozca el derecho a la presencia de los otros (familiares) y del paciente en los lugares de acogida.

PALABRAS CLAVE: reanimación cardiopulmonar presenciada, presencia familiar, reanimación cardiopulmonar

\section{Cardio-pulmonary Resuscitation Witnessed in the Mexican Hospital Context: a Study of Case in Patients and Relatives \\ In this article we study the witnessed cardio-pulmonary resuscitation (RCPP) in the context of the current cultural reconfigurations. From the opinion of 90 patients that entered Emergency Room and 90 relatives that accepted to participate in this survey, we found cultural favorable consensus toward the RCPp. This experience is rescued starting from a look that goes beyond the technical rationality, to establish some basis that can give origin to humanistic politics to recognize the right to the presence of the relatives in the admission places.}

KEYWORDS: witnessed cardio-pulmonary resuscitation, family presence and cardio-pulmonary resuscitation

Javier EdUaRdo G arcía de Alba G arCía: Unidad de Investigación Social Epidemiológica y en Servicios de Salud, IMSS-Jalisco, México javier_91046@yahoo.com

JuAn Francisco García Regalado: Hospital de Sub Zona 10 del IMSS, Guanajuato, México clanseta@yahoo.com

ANA L. SALCEDO Rocha: Unidad de Investigación Social Epidemiológica y en Servicios de Salud, IMSS-Jalisco, México analeticia_salcedorocha@yahoo.com.mx 
En una sociedad en que se naciera y muriera en casa, o en que el lisiado y el idiota no fueran desterrados de la plaza pública, en que se supiera distinguir la vocación médica de la profesión de plomero, se encontrarían personas para ayudar a los demás a vivir, sufrir o morir.

Ivan Ilich

\section{ANTECEDENTES}

S egún Phillippe Aries, hacia la mitad del siglo Xx la imagen tradicional de la muerte comenzó a experimentar mutaciones profundas en casi todas las sociedades occidentales, a tal grado que en el siglo XXI el morir ha llegado a ser literalmente innombrable. La mayoría de las instituciones ahora son lugares donde se ejerce una competencia técnica, un saber hacer cada vez más técnico y más eficaz, pero donde las cuestiones propias del sentido, las que se refieren a la subjetividad de los cuidadores y sus enfermos no tienen un lugar preciso (Duch y Mélich, 2005).

Una de las técnicas para salvar la vida de personas que presentan un paro cardiaco o respiratorio es la reanimación cardiopulmonar $(\mathrm{RCP})^{1}$, práctica conocida para quienes trabajan en el área de Urgencias de las instituciones de salud. Cuando se realiza la RCP en los nosocomios tradicionales, al igual que en otros procedimientos hospitalarios ${ }^{2}$, se pide a los familiares de los pacientes que se retiren "para que el equipo de salud pueda trabajar". Excluir a los familiares se justifica bajo la premisa de que los procedimientos invasivos y la agresión durante la reanimación pueden provocar estrés en los miembros de la familia y que su presencia podría comprometer el desempeño del equipo médico (Robinson et al., 1998).

La modalidad presenciada de la reanimación cardiopulmonar (RCPp) se realiza a partir de 1984 en el Hospital

\footnotetext{
${ }^{1}$ La RCP es un conjunto de maniobras mecánicas y eléctricas y procedimientos farmacológicos que realiza el médico con su equipo y que tienen como fin restablecer las funciones básicas del corazón y del pulmón para poder revertir la muerte clínica. La parte presencial implica el desarrollo de estas maniobras en presencia de un familiar, o sea, la aplicación del procedimiento habitual de reanimación frente a los familiares del paciente.

${ }^{2}$ Como la venopunción para obtener muestras de laboratorio, aplicar una solución intravenosa, cambiar un catéter, un lavado gástrico, etcétera.
}

Foot de Michigan, cuando el procedimiento y la actitud médica tradicional ante los familiares del paciente fueron cuestionados por el personal hospitalario, después de dos eventos en que los familiares pidieron estar presentes. Esta situación les permitió a los familiares participar con el equipo médico en el desarrollo del procedimiento. Desde entonces, esta nueva política hospitalaria ha provocado un continuo debate ${ }^{3}$ (Meyers et al., 1998).

La polémica en torno a la RCPp se presenta principalmente al interior del equipo de salud, y empieza a trascender fronteras y a dar como resultado la modificación y la división de las opiniones en Canadá, Estados Unidos y, muy recientemente, en México (García de Alba y García Regalado, 2007). La actitud conservadora y negativa hacia la RCPp ha sido más frecuente entre los médicos que en el cuerpo de enfermería.

Este debate ha involucrado a agrupaciones de profesionales, como la Asociación de Enfermeras de Urgencias y la Asociación Americana de Cuidados Críticos, que han pedido a los hospitales que autoricen la RCPp (Samples Twibell et al., 2008). Por su parte, la American Heart Association (AHA) en un principio se pronunció, en su manual ACLS (Advanced Cardiac Life Support) versión 2000, por permitir la presencia de la familia ${ }^{4}$ durante los intentos de reanimación "por ser una práctica clínica favorable, basada en la evidencia positiva que muestra el firme efecto terapéutico" (AHA, 2002). Sin embargo, en sus lineamientos de 2005 no se ratifica este procedimiento (AHA, 2005a y 2005b).

\footnotetext{
${ }^{3}$ El debate sobre la ayuda a los moribundos, así como el de la eutanasia, el suicidio asistido y el alargamiento de la vida se han convertido en temas candentes que dan entrada a posiciones radicales, opuestas y excluyentes. En el marco del proceso de industrialización-urbanización, agudizado por la transición demográfica, esto ha provocado la deconstrucción del escenario tradicional de morir. Así, el actual lugar del fallecimiento (casi siempre centros hospitalarios) presenta las características que Marc Augé atribuye a los no lugares; es decir, espacios que no pueden definirse ni como de identidad ni relacionales, ni históricos, un lugar de exilio aséptico y neutral, estructuras de acogida humanamente no significativas que diluyen el lugar como parte del proceso de socialización de los miembros de una sociedad (Duch y Mélich, 2005).

${ }^{4}$ Es un hecho ampliamente verificable (en el llamado "mundo occidental") que cada vez menos seres humanos mueren en sus casas rodeados por los miembros de su familia.
} 


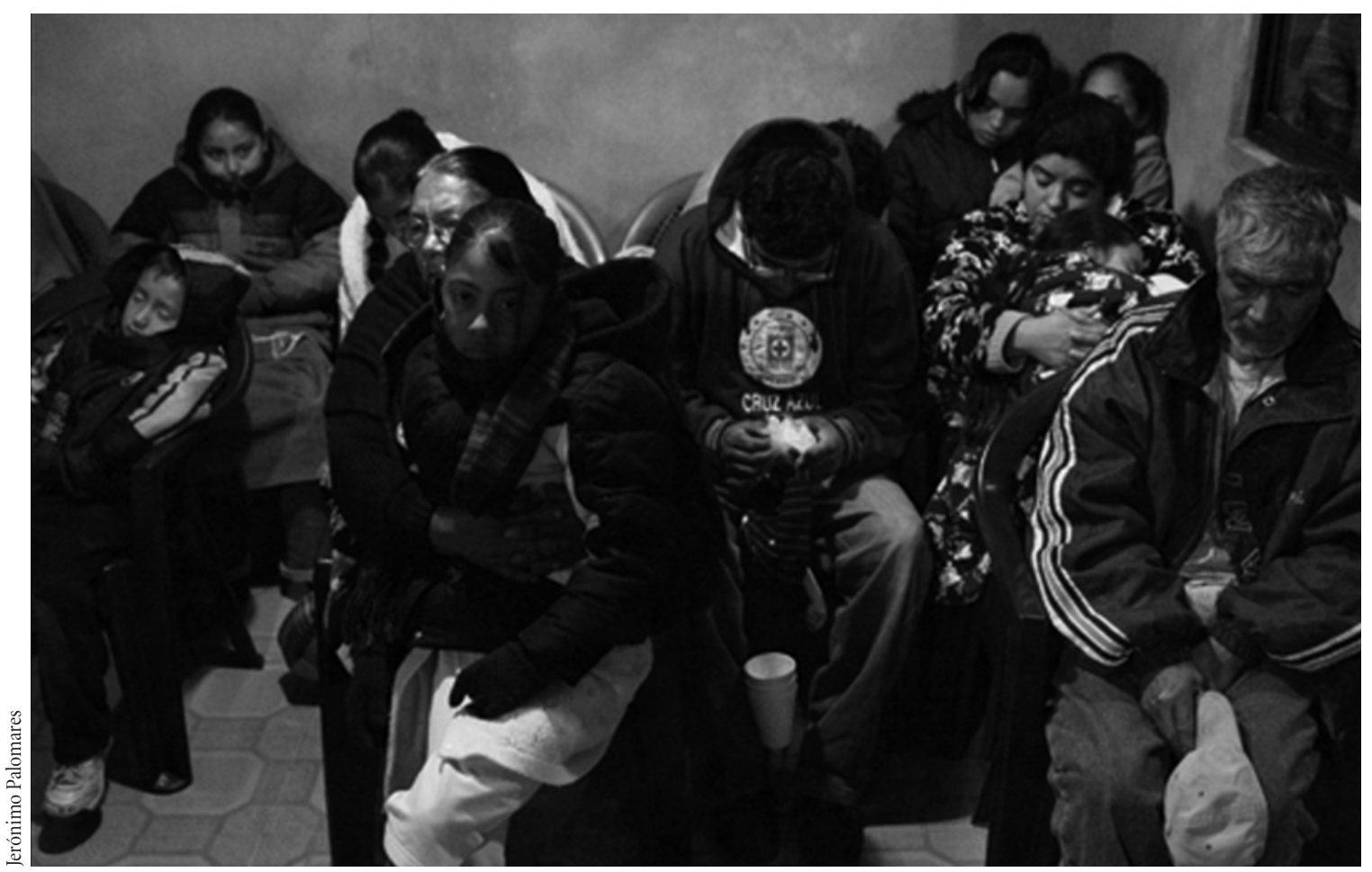

Sala de espera, 2008.

\section{ESTUDIOS RELACIONADOS}

En los últimos 15 años, en la literatura anglosajona se han realizado pocos trabajos sobre el tema. Halm (2005), en una revisión crítica, menciona la existencia de:

Veintidós estudios descriptivos en pacientes, familiares, o staff hospitalario, que señalan:

a) La opinión de los familiares, quienes arguyen que es su derecho estar presentes durante la resucitación, ya que piensan que su presencia ayuda tanto al paciente como a ellos mismos. No hay reportes de litigios judiciales por esta práctica.

b) La aceptación del personal de enfermería versus la opinión de los médicos, quienes argumentan que la presencia de familiares durante la RCP es disruptiva para el trabajo del equipo de salud, que no hay sustento científico para esta práctica y que la resucitación resulta traumática para los legos.
Dos estudios experimentales, donde:

a) No se encuentra evidencia de efectos psicopatológicos diferenciales entre los familiares presentes y los no presentes durante la RCP. Sin embargo, se concluye que la decisión de estar presente produce tranquilidad y satisfacción.

b) La educación incrementa el número de enfermeras que consideran que la RCPp es participativa y satisface una necesidad de cuidado en la atención al paciente en estado crítico.

c) Se plantean como elementos en contra de la RCPp: lo reducido de los espacios donde se realiza la práctica, la necesidad de proteger la privacidad de los pacientes, la disminución de la acción de apoyo del equipo biomédico, el miedo del equipo a enfocarse más a las necesidades de los familiares que a las de los pacientes y las posibles reacciones de los familiares durante el procedimiento. 
Los 24 estudios mencionados se realizaron bajo la perspectiva del paradigma cuantitativo.

Cuatro estudios cualitativos:

a) Sobre la experiencia de los pacientes, quienes manifestaron la importancia de la unión familiar con frases como: "se mantiene una conexión entre ellos y nosotros sus familiares"; "es reconfortante recibir apoyo y ayuda de todos", estar presentes en la RCP "es un derecho de los familiares", "nosotros siempre estamos juntos, hacemos todo juntos", "hacemos un buen equipo".

b) Los miembros de la familia, entre seis y 12 meses después de la RCP, señalan la importancia de la presencia en términos de: "estar conectados con el paciente" y de que el enfermo "sabía que los familiares estaban ahí en el momento de la RCP".

c) En cuanto a las perspectivas del equipo de salud, se encontraron tres opiniones. Tradicional: "lo importante es salvar la vida del paciente"; bifurcada: "lo importante es atender la vida del paciente y dar atención a su familia"; holística: "la sobrevida del paciente es lo principal, también es importante informar a la familia y ayudarlos a mantenerse activos".

Cinco estudios de tipo ético:

Se incluyen estudios de orden teleológico (beneficio para el paciente) y deontológico (sobre obligaciones y consecuencias morales para el equipo de salud).

\section{LOS ARGUMENTOS DEL DEBATE}

Los argumentos esgrimidos en contra y a favor de la RCPp pueden resumirse como sigue.

En contra:

1) La mayor parte de los estudios se basan en la medición de percepciones (Samples Twibell et al., 2008).

2) La falta de un marco teórico conceptual objetivo (Samples Twibell et al., 2008).

3) La inclusión de enfermeras de Urgencias como único universo de estudio (Samples Twibell et al., 2008).
4) El público está influido por las películas y los programas de televisión, donde dos terceras partes o más de los casos de RCP tienen un final feliz, pero en la realidad sólo 15\% de los casos tiene éxito (New Yorker, 2006).

5) La RCPp es un movimiento que tiene que ver con los intereses de las enfermeras y de los capellanes (New Yorker, 2006).

6) La RCPp va en contra del control médico de la atención a la vida y la muerte, es como si los pasajeros de un avión estuvieran en la cabina del piloto durante una emergencia (New Yorker, 2006).

7) Falta investigación con evidencias cabales sobre los beneficios de la RCPp (New Yorker, 2006).

8) En Estados Unidos sólo 5\% de los hospitales tienen políticas escritas sobre la RCPp (New Yorker, 2006).

A favor:

1) La forma de la medición de percepciones. Los trabajos actuales se plantean en términos de validez y confiabilidad estadística de los instrumentos aplicados (Samples Twibell et al., 2008).

2) Originalmente se trabajó con marcos teóricos implícitos; en la actualidad se utilizan teorías como la de difusión de la innovación de Rogers, la del cambio de Bandura, la fenomenología, etcétera (Samples Twibell et al., 2008).

3) Los trabajos de los últimos años incluyen al personal médico y de enfermería, así como a pacientes y familiares, no sólo del área de Urgencias sino de todo el hospital (García Regalado, 2007).

4) En otros estudios se ha encontrado que hasta en $47 \%$ de los casos llevados a las salas de Urgencias para pacientes críticos, los familiares estuvieron con el paciente durante el manejo de la emergencia. Ésta se inició en general en el ámbito prehospitalario, donde los propios familiares asistieron, ayudaron o dieron los primeros auxilios (Meyers et al., 1998).

5) Todos los familiares están agradecidos por poder presenciar y ayudar a su pariente en la reanimación (Booth et al., 2004). No ha habido demandas judiciales (Samples Twibell et al., 2008; Eichhorn et al., 2001).

6) La RCPp puede ser una oportunidad, tanto para el equipo médico como para los familiares, de entender y afrontar de manera compartida lo irracional y lo racional de 
la situación, y atenuar o dignificar el duelo (García Regalado, 2007).

7) El reverendo Hank Post (capellán) señala: "Si los familiares pueden estar presentes en el inicio de la vida de uno de sus integrantes, ipor qué no al final de su vida?” (New Yorker, 2006).

8) En el hospital fallece $54 \%$ de los pacientes crónicos, críticamente enfermos y ancianos, sin incluir a los que ingresan con patología aguda (debutantes de evento coronario, traumatizados, etc.). Estos pacientes manifiestan, en general, que morir solos es su temor principal; de ahí que el estar acompañado cobre un sentido y un significado relevante en la praxis del equipo de salud (Lynn et al., 1997).

9) Excluir a los familiares durante la RCP no es una política apropiada, toda vez que se ha demostrado objetivamente que aquellos que presenciaron la reanimación padecían menos estrés, recurrieron en menor medida a imágenes introspectivas y afrontaron mejor el duelo (Robinson et al., 1998).

\section{LA MUERTE EN LA ACTUALIDAD}

El proceso de medicalización y de deshumanización de la muerte en las sociedades modernas, donde priva el individualismo y la globalización, no plantea la existencia de marcos de sentido fijos, unívocos y estables, sino de diversos patrones de interpretación que son insuficientes para manejar el miedo y la inseguridad ante la muerte, aunque en ellos siguen figurando las tradiciones y las explicaciones religiosas.

En este contexto, la institución hospitalaria arranca al enfermo del seno de la familia y se hace cargo no del enfermo sino de su mal: un objeto aislado de su cotidianidad y transformado, como objeto científico y lingüístico, por los técnicos de la salud, quienes consideran la muerte un fracaso del cual no se puede hablar. Hay que guardar silencio ya que cualquier defunción, dentro de su racionalidad, es innominable, aspecto que se refleja en la relación médico-paciente-familiares (De Certau, 2000).

En este proceso de continua deconstrucción de la muerte, el morir ha sido conducido a un callejón sin salida, en el que los moribundos se encuentran atrapados, sin posibilidades de una muerte digna y humanamente significativa (Duch y Mélich, 2005), en un espacio donde la ciencia, la política y el pensamiento mágico compiten entre sí, prometiendo beneficios sin esfuerzo que en última instancia provocan graves contradicciones. La RCPp pone en evidencia estas contradicciones, las cuales podrían, en un momento dado, ser superadas mediante un tratamiento más humano del evento donde está en juego la vida de un ser querido.

\section{LA RCPp EN MÉXICO}

En nuestro país, los trabajos sobre RCPp son mínimos y aún no se ha establecido una política en relación con la aplicación de esta práctica (García Regalado, 2007). Esto puede ser consecuencia de una racionalidad conservadora que afecta al campo médico, tal como quedó reflejado en los resultados de un estudio previo realizado en México, según los cuales gran parte del personal médico y, en menor proporción, del grupo de enfermería que labora en las áreas de Urgencias no acepta la RCPp. Sin embargo, los motivos aludidos fueron diferentes, ya que los primeros creen que para los familiares es traumático presenciar la reanimación, mientras que las enfermeras consideran que el principal obstáculo es la falta de preparación de las familias. Así, se mostró que no existe acuerdo entre el grupo médico y el de enfermería para desarrollar la RCPp (García de Alba y García Regalado, 2007), situación que manifiesta una tensión político-axiológica en sus argumentos (Booth et al., 2004; Mc Clenathan et al., 2002). Por su parte, la enfermería ha transformado sus esquemas de pensamiento, al desarrollar el proceso enfermero sobre bases teóricas que se enfocan, de manera holística, en las necesidades de atención y de autoatención de los pacientes y de sus familiares, lo que implica una ampliación de horizontes y espacios teórico-prácticos en este campo.

Retomando a Halm (2005), Mc Clenathan et al. (2002) y Eichhorn et al. (2001), además de las opiniones de los protagonistas del equipo de salud hace falta conocer las voces del paciente y de los otros implicados: la familia. Por este motivo realizamos el presente trabajo, para tra- 


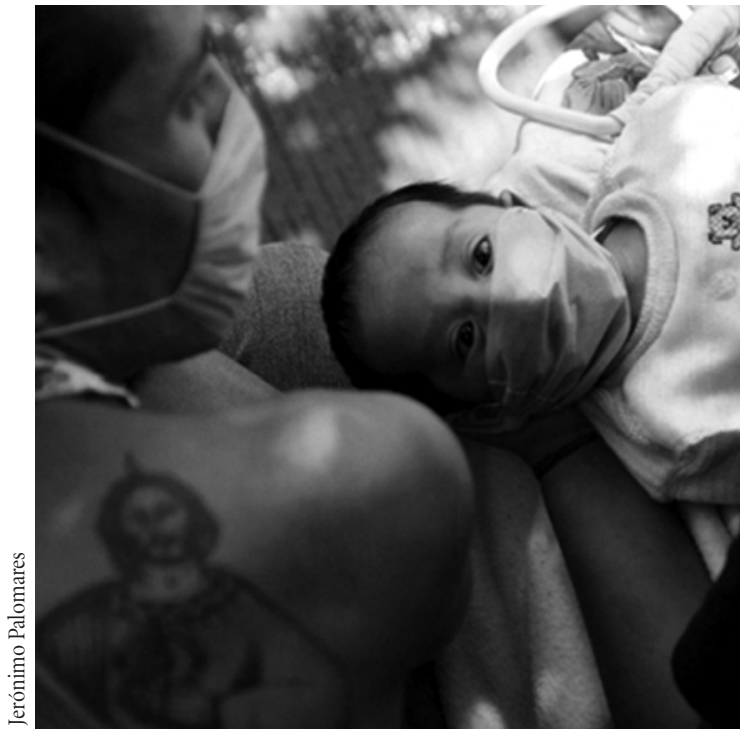

Sin título, 2009.

tar de ayudarnos a vislumbrar un poco mejor la cuestión de la RCPp. Resulta importante abordar estos acontecimientos en las salas de Urgencias de nuestro país desde la antropología médica; establecer los sentidos, significados, actitudes, creencias y prácticas de los pacientes y sus familiares respecto de su participación en la RCPp, ya que ésta puede considerarse, más que un indicador de calidad de los servicios de salud ${ }^{5}$ (Huerta Torrijos et al., 2001; Warren, 1989), un indicador de la salud de dichos servicios, al hacer que los ámbitos donde se produce la muerte sean humanamente significativos y simbólicamente activos; es decir, estructuras de acogida que sean capaces de transmitir adecuadamente la gramática de los sentimientos (Duch y Mélich, 2005), sin que sea secuestrada la experiencia por imposición de una cultura que suprime los campos de la moral y la ética por la expansión del conocimiento técnico (Giddens, 1997: 205-210). Así, el objetivo del presente estudio es explorar la opinión de los pacientes y sus familiares respecto de estar acompañado o acompañar a su familiar en episodios de RCPp.

\footnotetext{
${ }^{5}$ Desde este punto de vista parecería que la praxis médica ha llegado a la conclusión de que por la simple aplicación de los últimos avances tecnológicos de la medicina a las supuestas causas de la enfermedad, ya resulta posible el aniquilamiento de la misma muerte.
}

\section{ESTUDIO EN EL SERVICIO DE URGENCIAS}

Se realizó un estudio de caso durante el último semestre del año 2006, que incluyó una muestra propositiva ${ }^{6}$ de 180 participantes — 90 pacientes y 90 familiares—, elegidos aleatoriamente de la lista de ingreso de pacientes a Urgencias, e incluidos en forma consecutiva. Todos, asistentes al servicio de Urgencias del Hospital General de Zona núm. 46 del Instituto Mexicano del Seguro Social (IMSS) en Guadalajara, México. Se incluyó a los pacientes que tenían más de 18 años, que consintieran informadamente $^{7}$ participar en el estudio ${ }^{8}$ y que tuvieran un tiempo de hospitalización en la Sala de Urgencias mayor de cuatro horas, sin importar la patología que condicionara su ingreso. Entonces, fueron excluidos los menores de 18 años, quienes por su gravedad ameritaban menos de cuatro horas de hospitalización y aquellos cuyo estado mental estaba alterado por efecto de medicamentos, drogas o estado de coma. Tampoco se incluyó a pacientes que se encontraban en una condición que pusiera en peligro inmediato su vida y que requirieran maniobras inmediatas de reanimación, así como a aquellos cuyo deterioro progresivo del estado de salud pusiera en riesgo su vida en corto plazo. Los criterios de ingreso para los familiares fueron ser mayor de 18 años y familiar acompañante del paciente, y firmar una carta de aceptación de participación en el proyecto.

Se aplicó primero un cuestionario estructurado ( $A H A$, 2005b y Robinson et al., 1998) a cada uno de los 180 participantes. La primera parte del instrumento recolectó datos sociodemográficos, tales como edad, sexo, escolaridad, patología de ingreso, tiempo de evolución de la misma y nivel de ingreso económico. La segunda parte

\footnotetext{
${ }^{6}$ El tamaño de la muestra cubre sobradamente lo recomendado por Weller y Rommey (1988); 28 casos mínimo para 95\% de confianza y $90 \%$ de potencia, tomando como acuerdo cultural un valor de 50 por ciento.

${ }^{7}$ Se les pidió que contestaran a la siguiente pregunta: en el supuesto de que su corazón se detuviera y tuviéramos que ayudarle para que volviera a trabajar, ¿le gustaría que estuviera presente algún o algunos familiares? En el caso de los familiares, el supuesto se planteó así: en caso de que su familiar tuviera un paro y se necesitara hacer maniobras para revivirlo, ¿le gustaría estar en la habitación donde se realizan las maniobras?

${ }^{8}$ No hubo rechazos para participar.
} 
del estudio incluyó la aplicación de nueve reactivos, propuestos en forma previa por Meyers (Meyers et al., 1998), para determinar la opinión de los encuestados acerca de la reanimación presenciada, utilizando como medio de evaluación respuestas dicotómicas (sí o no). Como tercera parte del estudio, se solicitó a los participantes un listado de conceptos (listas libres ${ }^{9}$ ) para las nueve preguntas aplicadas.

Los datos sociodemográficos y de opinión se procesaron a través de los programas EPIINFO 6.0 y SPSS 12.0 para obtener los estadígrafos descriptivos e inferenciales de acuerdo con el tipo de escala en que fueron expresadas las variables. El nivel de significancia estadística se determinó a partir de una p menor o igual a 0.05. El análisis de consenso sobre las diferentes situaciones propuestas en cada pregunta de las listas libres, para cada grupo, se inició estructurando una lista jerárquica de frecuencia de respuestas o modelo de ítems para la pregunta formulada. Estas respuestas individuales fueron codificadas para establecer matrices de correlación y aplicar el método de principales componentes con apoyo del programa Anthropac ${ }^{\circledR}$. La determinación de consenso cultural, como la coincidencia de acuerdo sobre el arreglo jerárquico (modelo de ítems) de las respuestas dadas por los informantes, se midió con base en la razón de variabilidad, ya que si ésta es mayor que 3 , entonces se considera que existe una diferencia significativa entre el arreglo consensado versus otros arreglos posibles. Eso nos indica que tenemos un modelo de arreglo semántico, que es compartido, único y que no está dado por el azar ( $\mathrm{p}<0.05)$ (Borgatti, 1992).

\section{GRUPOS PARTICIPANTESY SUS RESPUESTAS}

En el cuadro 1 (p. 157) se presentan las principales características sociodemográficas de los dos grupos participantes (pacientes y familiares). Se muestran diferencias estadísticas favorables al grupo de familiares según su composición por sexo (más mujeres), edad (más jóvenes), escolaridad (mayor nivel de estudios) e ingreso eco-

\footnotetext{
${ }^{9}$ La técnica es una forma estructurada de hacer preguntas, por ejemplo: Dígame usted, ¡cuáles son los motivos para estar de acuerdo con la RCPp?
}

nómico (mejor salario). Obsérvese que uno de cada tres pacientes ingresa por problemas quirúrgicos. En el cuadro 2 (p. 158) las opiniones de ambos grupos hacen patente diferencias estadísticamente significativas en dos de las nueve preguntas formuladas. Los familiares entrevistados se declaran más a favor sobre la ayuda que les proporcionaría a ellos mismos durante el proceso de duelo haber participado en la RCPp (pregunta 3 ) y sobre sentirse bien consigo mismos por haber estado presentes durante la RCPp (pregunta 4).

En las asociaciones entre las variables de los pacientes y las respuestas otorgadas, se encontró una correlación positiva (directamente proporcional) entre la edad del paciente con la respuesta favorable a que sus familiares presenciaran su RCP en el caso de necesitarla $(r=0.772$ y $\mathrm{p}<0.05)$. También se observó que los pacientes que presentaban otro padecimiento, aparte de su patología de ingreso a Urgencias, eran más proclives a considerar que los familiares deben permanecer con ellos antes del momento de la muerte ( $\mathrm{Ji}^{2}$ de $32.47, \mathrm{p}<0.001$ ). Para el grupo de los familiares, se encontraron correlaciones positivas entre la edad y el enfoque positivo del proceso de duelo, si su paciente llegara a fallecer $(r=0.761$ y $\mathrm{p}<0.05)$, y entre la edad y la creencia de que la población aún no se encuentra lista para poder presenciar la reanimación de sus enfer$\operatorname{mos}(\mathrm{r}=0.751$ y $\mathrm{p}<0.05)$.

En el mismo grupo de familiares se encontraron diferencias estadísticamente significativas al comparar las respuestas de los subgrupos con ingresos de menos de cuatro salarios mínimos y el de los que perciben cuatro salarios mínimos o más. Los últimos consideran que poder presenciar la reanimación de su ser querido les ayudaría a afrontar mejor su proceso de duelo en caso de que éste llegara a fallecer $\left(\mathrm{Ji}^{2}=8.3, \mathrm{p}<0.01\right)$.

En relación con el análisis de consenso de los dos grupos sobre la RCPp, los pacientes manifestaron acuerdo estadísticamente significativo en sus respuestas a las siguientes preguntas:

1) ¿Qué experiencia le dejó presenciar previamente algún(os) evento(s) de RCP?

El acuerdo compartido o consenso cultural (razón de variabilidad $14.5, \mathrm{p}<001$ ) se manifestó en torno a respuestas relacionadas con: 
a) Constatar que se hizo lo posible por conservar la vida del enfermo. V. gr.: "le hicieron toda la lucha hasta el último minuto".

b) Obtener elementos para una mejor aceptación del proceso de muerte. V. gr.: "así no tengo dudas", "murió acompañado", "se le ayudó para que muriera en paz".

c) Darse cuenta de que estar presente durante la RCP no es un proceso no exento de nerviosismo y angustia. $V . g r$.: "no es fácil", "se siente bien duro", "duele".

2) ¿La población general se encuentra lista o no para presenciar situaciones de RCP?

Se encontró un solo modelo de acuerdo significativo (razón de variabilidad 5.26, p $<0.05$ ) que incluyó tres tipos de respuestas:

a) Sí, porque de esa manera se le dan ánimos, tanto al paciente como a los familiares. V. gr: "porque sería la última vez que los veamos con vida", "porque así se le puede ayudar a él, para poder irse en paz", "para que no se sienta solo", "para despedirnos de él".

b) No, por varias razones. $V$. gr.: "estorbarían las maniobras de los doctores", "porque (algunos de los familiares) son cobardes y les dolería la situación por impactante".

c) No condicionado. V. gr.: "porque hace falta comunicación por parte del personal médico", "el personal del hospital no explica el proceso de RCP a los familiares".

Para el grupo de los familiares, se encontró consenso en sus respuestas a una sola pregunta (razón de variabilidad 3.43, $\mathrm{p}<0.05)$. Al preguntarles si los familiares deben acompañar a sus enfermos antes de morir, consideran como argumentos:

a) A favor: "porque los queremos", "porque somos sus familiares", "porque formamos parte de la misma familia”; señalan además que presenciarían la RCP sin disgusto; aseguran que "es nuestro deber", "para corroborar que los médicos hacen lo que está indicado", "para ver que se hizo la lucha hasta el final".

b) En contra: no querrían estar porque creen que son cobardes y que les dolería, podrían ponerse nerviosos y no desearían ver sufrir a su familiar.

c) En todos los casos se planteó un sentimiento de apoyo hacia el enfermo moribundo a pesar de que a ellos les pudiera ocasionar sentimientos incómodos. $V$. gr.: "es traumante", "no me gusta ver sufrir a nadie". Consideraron que la experiencia finalmente sería satisfactoria. V. gr: "estar ahí me ayudaría en caso de que muriera, por lo menos para decirle adiós y que no se sienta solo".

\section{ALTERNATIVAS FRENTE A LA MUERTE}

Una fortaleza de este estudio es ofrecer un tema novedoso que invita a reflexionar sobre el quehacer del equipo de salud en un asunto que ha sido un tanto esquivado por tocar la etapa final de la vida humana y tener implicaciones de diverso orden. Como principal restricción, el trabajo debe circunscribirse en sus resultados al ámbito y a los grupos analizados, pacientes y familiares asistentes al servicio de Urgencias del Hospital de Zona núm. 46 del IMSs de Guadalajara, México.

\section{EL CONTEXTO DE URGENCIAS}

No debe perderse de vista que el área de Urgencias hospitalarias es una zona de conflicto, de caos organizado. Es una heterotopía controlada por expertos que concentran y fomentan la tecnología médica, un espacio donde hay que tomar decisiones de forma rápida, circunstancia que "justifica" el "aislamiento" de los pacientes, descalificados para participar en actividades sociales ortodoxas, los cuales son atendidos en una relación médico-paciente autoritaria, dada su delicada o grave condición. Dentro de esta racionalidad técnica, la acción médica no puede esperar - sobre todo en procedimientos como la RCPp - para atender un paro cardiaco o respiratorio, cuando la oportunidad de intervención marca la diferencia entre la vida y la muerte.

Sin embargo, la rápida intervención médica puede provocar errores de actuación, miedo o subvaloración de los sentimientos y significados de los otros. Por esta razón, la forma más común de evadir conflictos con los familiares es la exclusión física, la distancia sociocultural, el alejamiento, la negación que hace tabla rasa de todo 
Cuadro 1. Características de los participantes

\begin{tabular}{|c|c|c|c|c|c|}
\hline \multirow{2}{*}{$\begin{array}{l}\text { Variable } \\
\text { Género }\end{array}$} & \multicolumn{2}{|c|}{$\begin{array}{c}\text { Grupo } \\
\text { de pacientes } \\
n=90\end{array}$} & \multicolumn{2}{|c|}{$\begin{array}{c}\text { Grupo } \\
\text { de familiares } \\
n=90\end{array}$} & \multirow[t]{2}{*}{ Observaciones } \\
\hline & Número & Porcentaje & Número & Porcentaje & \\
\hline Masculino & 37 & 41 & 15 & 17 & \multirow{2}{*}{$\begin{array}{l}\mathrm{Ji}^{2}=13.09 \\
\mathrm{p}=0.0002\end{array}$} \\
\hline Femenino & 53 & 59 & 75 & 83 & \\
\hline \multirow[t]{2}{*}{ Edad } & Promedio & $\begin{array}{l}\text { Desviación } \\
\text { estándar }\end{array}$ & Promedio & $\begin{array}{c}\text { Desviación } \\
\text { estándar }\end{array}$ & \multirow[t]{2}{*}{$\begin{array}{l}\mathrm{T}=1.90 \\
\mathrm{p}=0.05\end{array}$} \\
\hline & 50.7 & 17.41 & 46.02 & 15.65 & \\
\hline Escolaridad & Número & Porcentaje & Número & Porcentaje & \\
\hline Analfabetas & 18 & 20 & 7 & 8 & \multirow{6}{*}{$\begin{array}{l}\mathrm{Ji}^{2}=25.75 \\
\mathrm{p}=0.000002\end{array}$} \\
\hline Primaria & 39 & 43 & 31 & 34 & \\
\hline Secundaria & 19 & 21 & 21 & 23 & \\
\hline Preparatoria & 7 & 8 & 7 & 8 & \\
\hline Carrera técnica & 5 & 6 & 8 & 9 & \\
\hline Profesional & 2 & 2 & 16 & 18 & \\
\hline Parentesco & & & Número & Porcentaje & \\
\hline Hijo(a) & & & 30 & 33 & \\
\hline Esposo(a) & & & 25 & 28 & \\
\hline Padre/Madre & & & 15 & 17 & \\
\hline Hermano(a) & & & 5 & 6 & \\
\hline Nieto(a) & & & 4 & 4 & \\
\hline Abuelo(a) & & & 2 & 2 & \\
\hline Otro & & & 9 & 9 & \\
\hline Ingreso económico & Número & Porcentaje & Número & Porcentaje & \\
\hline$<1$ salario mínimo & 12 & 13 & 14 & 15.55 & $\mathrm{Ji}^{2}=10.89$ \\
\hline 1 salario mínimo & 30 & 33.5 & 15 & 16.66 & $\mathrm{p}=0.01$ \\
\hline $\begin{array}{l}\text { 2-4 salarios } \\
\text { mínimos }\end{array}$ & 30 & 33.5 & 26 & 28.88 & \\
\hline $\begin{array}{l}>4 \text { salarios } \\
\text { mínimos }\end{array}$ & 18 & 20 & 35 & 38.88 & \\
\hline Patología & Número & Porcentaje & & & \\
\hline Quirúrgica & 32 & 35 & & & \\
\hline Médica & 58 & 65 & & & \\
\hline
\end{tabular}


Cuadro 2. Opinión sobre preguntas formuladas

\begin{tabular}{|c|c|c|c|c|c|}
\hline \multirow{3}{*}{ Pregunta } & \multicolumn{2}{|c|}{ Grupo de pacientes } & \multicolumn{2}{|c|}{ Grupo de familiares } & \multirow{3}{*}{ Observaciones } \\
\hline & \multicolumn{4}{|c|}{ Porcentaje } & \\
\hline & Sí & No & Sí & No & \\
\hline $\begin{array}{l}\text { 1) ¿Cree que los familiares deberían es- } \\
\text { tar con los enfermos justo antes de la } \\
\text { muerte? }\end{array}$ & 90 & 10 & 81 & 19 & $\begin{array}{l}\mathrm{Ji}^{2}=2.55 \\
\mathrm{p}=0.11\end{array}$ \\
\hline $\begin{array}{l}\text { 2) ¿Si tuviera la oportunidad de que } \\
\text { sus familiares estuvieran en el mismo } \\
\text { cuarto que usted mientras lo reaniman, } \\
\text { ¿piensa que le podría ayudar en ese mo- } \\
\text { mento? }\end{array}$ & 63 & 37 & 51 & 49 & $\begin{array}{l}\mathrm{Ji}^{2}=2.43 \\
\mathrm{p}=0.11\end{array}$ \\
\hline $\begin{array}{l}\text { 3) ¿Si tuvieran la oportunidad, ¿cree que } \\
\text { el estar ahí le ayudaría a sus familiares } \\
\text { con el sentimiento de pérdida y tristeza } \\
\text { luego que usted muera? }\end{array}$ & 67 & 33 & 45 & 55 & $\begin{array}{l}\mathrm{Ji}^{2}=8.59 \\
\mathrm{p}=0.003\end{array}$ \\
\hline $\begin{array}{l}\text { 4) Si sus familiares tuvieran la opor- } \\
\text { tunidad de estar en el cuarto al mo- } \\
\text { mento de reanimarlo, ¿cree que ellos } \\
\text { se sentirían bien? }\end{array}$ & 63 & 37 & 37 & 63 & $\begin{array}{l}\mathrm{Ji}^{2}=11.37 \\
\mathrm{p}=0.0007\end{array}$ \\
\hline $\begin{array}{l}\text { 5) Si llegado el caso te preguntaran si } \\
\text { quisieras que tus familiares estuvieran } \\
\text { en tu reanimación, ¿rechazarías esta } \\
\text { decisión? }\end{array}$ & 37 & 63 & 43 & 57 & $\begin{array}{l}\mathrm{Ji}^{2}=0.74 \\
\mathrm{p}=0.38\end{array}$ \\
\hline $\begin{array}{l}\text { 6) ¿Has tenido alguna experiencia pre- } \\
\text { via con algún familiar que necesite que } \\
\text { se le reanime? }\end{array}$ & 25 & 75 & 26 & 74 & $\begin{array}{l}\mathrm{Ji}^{2}=0.00 \\
\mathrm{p}=0.98\end{array}$ \\
\hline $\begin{array}{l}\text { 7) ¿Consideras que la población se en- } \\
\text { cuentra preparada para esta práctica? }\end{array}$ & 15 & 85 & 19 & 81 & $\begin{array}{l}\mathrm{Ji}^{2}=0.32 \\
\mathrm{p}=0.57\end{array}$ \\
\hline $\begin{array}{l}\text { 8) ¿Consideras que los médicos se en- } \\
\text { cuentran preparados para aceptar este } \\
\text { tipo de práctica? }\end{array}$ & 43 & 57 & 45 & 55 & $\begin{array}{l}\mathrm{Ji}^{2}=0.01 \\
\mathrm{p}=0.92\end{array}$ \\
\hline $\begin{array}{l}\text { 9) ¿Consideras que presenciar tu rea- } \\
\text { nimación pudiera ser traumático para } \\
\text { tu familia? }\end{array}$ & 50 & 50 & 64 & 36 & $\begin{array}{l}\mathrm{Ji}^{2}=3.00 \\
\mathrm{p}=0.07\end{array}$ \\
\hline
\end{tabular}

Fuente: Elaboración propia.

sentimiento, evitando así cualquier transferencia entre el equipo de salud y el paciente y sus familiares.

Si a esto se le agrega que la mayoría de las muertes ocurridas en el Servicio de Urgencias se presenta de manera súbita, el proceso de afrontamiento de los actores es muy diferente al que sucede en el área de hospitalización, donde se atiende a otro tipo de pacientes con enfermedades crónicas.
Al ocurrir una defunción en Urgencias, la familia afronta la pérdida con un dolor agudo, difícil de procesar de manera inmediata, porque se preguntan ¿cómo su familiar que entró vivo, y a veces caminando, sale muerto?, o ¿cómo entender que el joven salió de casa bien y ahora lo reportan en un estado agónico? Ahí es donde consideramos que la RCPp, a pesar de que a menudo se maneja como un problema técnico, puede ofrecer a los actores una oportu- 
nidad de afrontamiento y entendimiento de la situación de desesperanza, útil para mitigar el duelo y generar sentimientos reconfortantes.

En el hospital estudiado, como en los demás nosocomios mexicanos ${ }^{10}$, usualmente el familiar se adapta a las reglas institucionales (Duch y Mélich, 2005), al amparo de las cuales se saca al familiar de la habitación, bajo el supuesto de que "no saben lo que va ocurrir" o de "protegerlos de situaciones traumáticas"; sin embargo, como señalan Marguntyen y sus colaboradores (2005), suponer lo que ocurre u ocurrió puede ser igual o peor de desesperante que haberlo visto, lo que además hace desaparecer la incertidumbre. La política de exclusión física y social de pacientes y familiares incrementa la percepción del miedo en ambas partes, convirtiendo al Servicio de Urgencias en un lugar de aislamiento y erosión de los vínculos personales (Olvera y Sabido, 2007), un lugar donde, paradójicamente, por un lado la muerte se maneja como una magnitud abrumadora, difícil de pensar, y por el otro como un problema técnico-científico al margen de simbolismos y rituales; es decir, fuera de la racionalidad constitutiva del ser humano.

\section{LOS GRUPOS ESTUDIADOS}

En el grupo de los familiares, más joven que el de los pacientes, predominaron mujeres ${ }^{11}$ con mayor nivel socioeconómico, fenómeno comúnmente observado en los servicios de hospitalización mexicanos y que caracteriza el rol del acompañante (Martínez, 2007). Esta situación, que obviamente implica una perspectiva diferente en re-

\footnotetext{
10 Tradicionalmente, se temía morir súbitamente sin auxilios espirituales para salvar el alma, función básica del antiguo hospital de caridad. En la actualidad la muerte se ha conformado en una experiencia permanente que varía según el tipo de sociedad y el marco de significados que le atribuyen un sentido, siendo el (los) miedo(s) una de las vías de unión —y de las más importantes- a través de las cuales fluye la estructura de la sociedad sobre las funciones psíquicas individuales (Elías, 1994: 527). Sentir miedo a la muerte es algo innato, pero su intensidad y tipo dependen de las relaciones sociales en las cuales el ser humano se encuentre inmerso, así como de las historias de las mismas (Olvera y Sabido, 2007).

${ }^{11}$ Sobre todo esposas e hijas.
}

lación con la del grupo de pacientes, explica algunas diferencias en las respuestas, en las que los familiares parecen justificar la RCPp en función de lo que ésta puede ayudarles a afrontar el duelo y a sentirse bien consigo mismos, en caso de fallecer su ser querido.

Las opiniones vertidas revelan que la tradición se mantiene: tanto los pacientes (90\%) como los familiares (81\%) manifestaron su deseo de que los últimos estén presentes antes de la muerte del paciente. Sin embargo, cuando se les pregunta sobre la ayuda de la presencia de familiares durante la RCPp, el porcentaje en ambos grupos se reduce a $67 \%$ y $45 \%$, respectivamente. El contraste de opiniones se torna estadísticamente significativo cuando los pacientes consideran que la RCP ayudaría a sus familiares a afrontar el duelo y que se sentirían bien durante la misma, mientras que los familiares señalan que la RCPp podría ser traumática para la familia.

Llama la atención que tres de cada cuatro de los participantes indicaran tener experiencia en el proceso de RCP, lo cual sugiere que se están mezclando la experiencia vivencial y la mediática ${ }^{12}$. Esto pudo influir en la opinión idealizada de pacientes y familiares en el sentido de que la población está preparada para la RCPp, lo cual contrasta con el caso de los médicos, que en menor porcentaje están preparados para aceptar la RCPp, quizás como consecuencia del conservadurismo que priva en el sector salud, donde el distanciamiento es la consigna que cuestiona la imposibilidad institucional de sostener la angustia, la desesperanza o el dolor.

El análisis de consenso pone de relieve la ambigüedad en el manejo de un concepto nuevo: la RCPp, donde por una parte privan la tradición y los sentimientos solidarios de amor familiar y el reconocimiento del miedo y del sufrimiento e inclusive de la evasión ante el proceso de morir del otro, y por otra parte el "respeto" ante la hegemonía médica y sus límites de acción en el hospital, el reclamo

\footnotetext{
12 Evidentemente la muerte no es un espectáculo, a diferencia de la muerte banal que ofrecen el cine, la televisión, el radio y la prensa como muerte informativa, es el desencadenante de emociones insoportables que "rompen" el equilibrio biopsicosocial de la vida cotidiana por eso hay que ocultar la muerte y a los moribundos de la sociedad (Dutch y Mélich, 2005).
} 


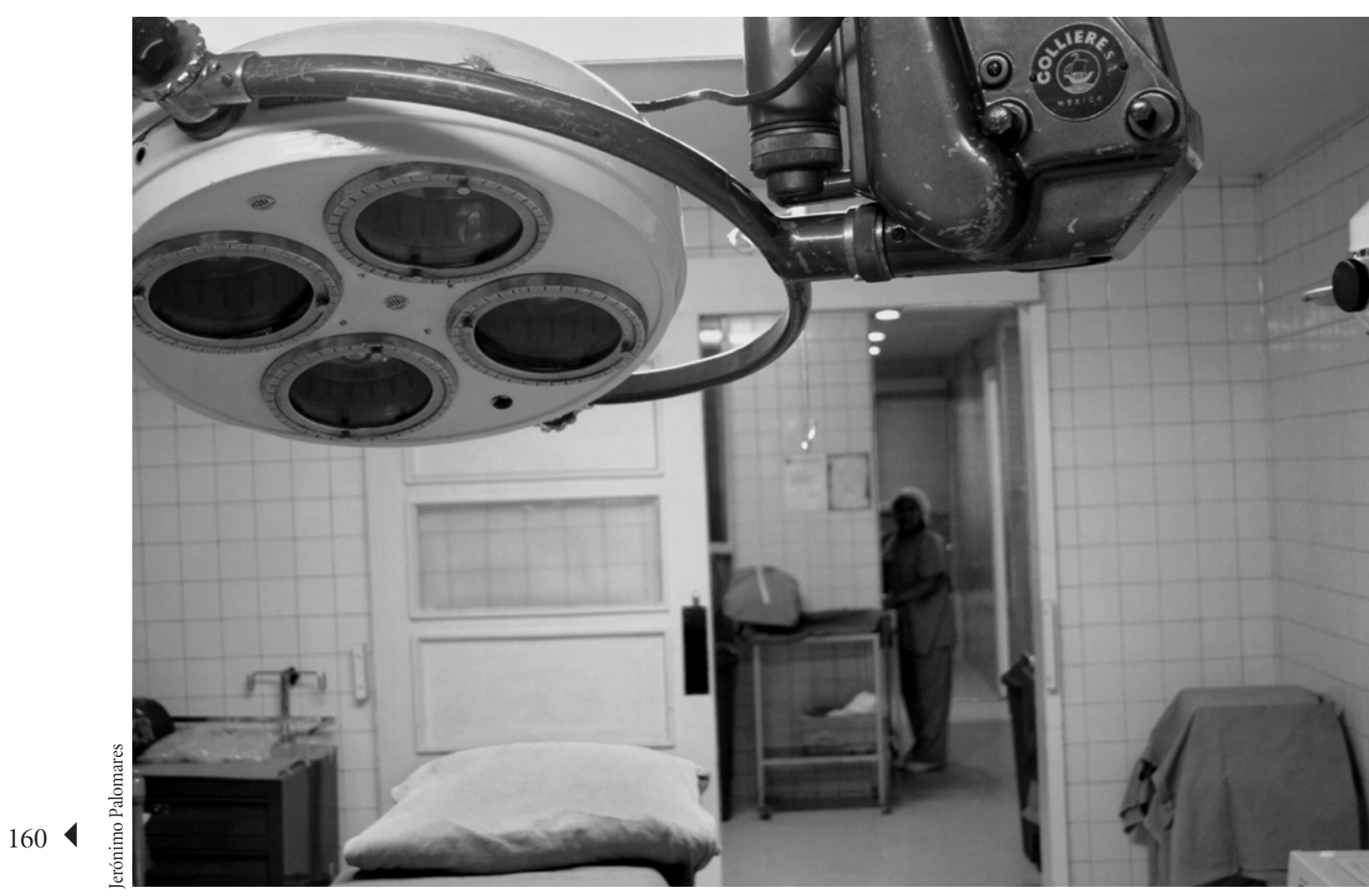

Sanatorio de San Hipólito, 2010.

ante la expropiación del ser querido, el manejo discrecional de la información y el silencio sobre el estado del paciente por parte del equipo de salud, que atenta contra los derechos y la necesidad de los familiares de constatar que todo se hizo "bien", dentro de lo posible.

La existencia de un modelo cognitivo de la RCPp, culturalmente compartido en pacientes y familiares, forma un marco explicativo con elementos a favor y en contra que deben ser tomados muy en cuenta por los servicios de salud, ya que pueden funcionar como guía o heurística para desarrollar acciones prácticas relacionadas con la transformación de los "lugares de aislamiento" en verdaderas estructuras de acogida, en espacios de consolidación social, donde pueda caber la promoción de la RCPp sin que ésta sea vista como una respuesta técnica a un "fallo mecánico" del cuerpo humano. Es decir, espacios que complementen el buen vivir con el buen morir.

\section{MORIRY RCPp}

El consenso y las respuestas dadas por familiares y pacientes ponen de manifiesto lo señalado por Gómez y Delgado (2000):

1) La muerte no es un asunto individual; también implica a los seres queridos, ya que es una vivencia compartida.

2) Morir siempre será difícil, ya que para que un ser humano muera con aceptación y dignidad necesita la ayuda humana en ese acto de transición pleno de simbolismos.

3) Tenemos que presenciar la muerte o vivir la muerte de otros para reconocer la muerte propia y la fragilidad de nuestra vida. 
El dilema de morir se acentúa al percibir una esperanza de vida con la aplicación de la RCPp, situación que desvía el sentido de los sentimientos de miedo-angustia, por ser la muerte una circunstancia esperada, injusta, democrática y, sobre todo, definitiva. La RCPp pospone la posibilidad de que nuestra individualidad desaparezca, que nuestro cuerpo se descomponga y que nuestra conciencia se diluya en el éter (Gómez y Delgado, 2000). Y aunque las nuevas generaciones de mexicanos tienen más información sobre la muerte y su prevención que las precedentes, lamentablemente han aumentado la violencia y la deshumanización de la vida y de la muerte. La RCPp no puede verse como una simple solución mecánica ante el desperfecto de la maquinaria humana, sino que tiene que concebirse de forma compleja, como la unión de la representación que se tiene de la enfermedad y de la muerte (castigo, destino, descuido, mala suerte, etc.) con la posibilidad de "curación" (realizada por Dios o por exper$\left.\operatorname{tos}^{13}\right)$, las relaciones, los deberes y la posición que ocupan los protagonistas (enfermos y familiares).

El deseo de los pacientes de que sus familiares estén presentes en el momento de la RCP concuerda con lo reportado por Mangurten et al. (2005) y Meyers et al. (2000), y puede interpretarse como una expresión del deseo de no estar solo ante la posibilidad de la muerte, combinación de miedos y deseos - como lo han reportado Lynn et al. (1997) y Eichhorn et al. (2001) — al encarar el momento, ya sea por el posible dolor, por no encontrarse preparado por estar aislado de sus seres queridos. A estas razones, nuestro estudio agrega elementos tales como la manutención de la unidad familiar, el acompañamiento simbólico y, de manera marginal, el asegurar el consuelo de que los pacientes fueran vistos por el equipo de salud como seres humanos.

La asociación de que a mayor edad existe más aceptación de la reanimación presenciada sugiere la posibilidad de un mayor contacto (real o virtual) con la práctica de la RCPp, pero sobre todo un riesgo de morir ascendente, situación que cobra grave significado. El hipotético bene-

\footnotetext{
${ }^{13}$ Según se acoja el demandante a la ciencia (despojada de religiosidad, pero conservadora de la moral) o a la religión (como garantía de una nueva y mejor vida).
}

ficio de participar en la RCPp declarado por los familiares es refrendado por los escritos de Mangurten et al. (2005), Meyers et al. (1998 y 2000), Robinson et al. (1998) y Rosenczeig (1998), quienes reportan menor ansiedad y depresión, así como sentimientos positivos en los familiares y hacia el personal de salud, siendo el motivo principal la sensación de haber hecho todo lo posible para salvar a su ser querido, y haber podido confortarlos y ser escuchados por ellos en sus últimos momentos. Beneficios notablemente superiores a los de haberse quedado sentados en la sala de espera ${ }^{14}$.

\section{ASPECTOS ÉTICOS}

No podemos dejar de señalar que la visión del paciente debe considerarse no sólo por razones disciplinares, sino también de ética y de humanidad, ya que esta mirada incluye vivencias previas, presentes y en gestación para la RCPp, como una forma de afrontar directamente la posibilidad de morir. En cambio, entre los familiares este sentido, aunque es en principio solidario con su ser querido, también remite hacia su propia persona: el sufrimiento como vivencia personal o como una situación hipotética de su propia muerte.

Lo anterior incide profundamente en la forma en que el personal de salud se prepara para enfrentar profesionalmente la muerte y polemiza la manera de abordarla, ya que excluir de las decisiones y del momento mismo a los familiares y alejarse objetiva y subjetivamente de los pacientes son motivo de cuestionamiento al hospital moderno, el cual es criticado por la crudeza de los planteamientos que hace el personal y por el aislamiento de los enfermos desahuciados, lo que se traduce en una muerte solitaria y mecánica. Pero es justo señalar que, a diferencia de Meyers et al. (2000), en nuestro estudio no encontramos como idea prioritaria la necesidad de los familiares de estar presentes para que los médicos no equivocaran su atención,

\footnotetext{
${ }^{14}$ La revisión de la literatura de los últimos años no muestra ninguna evidencia de que los familiares estorben o interfieran durante las maniobras de reanimación (AHA, 2005b; Booth et al., 2004; McClenathan et al., 2002; Mangurten et al., 2005; Lynch y Medin, 2006 y Moreland y Manor, 2005).
} 


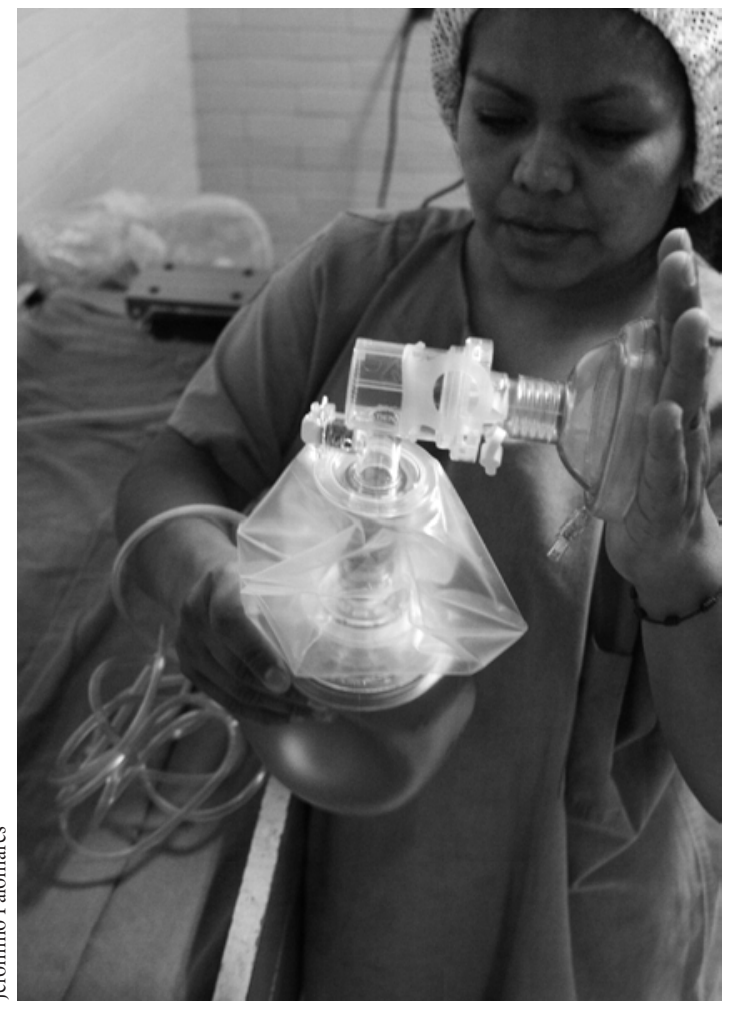

Sanatorio de San Hipólito, 2010.

sino más bien en el sentido de asegurar que sus pacientes recibieran un trato humano. Las ideas prioritarias fueron la solidaridad con su ser querido y el fortalecimiento de su yo ante la vicisitud de la muerte del paciente ${ }^{15}$. En ambos resultados, el trasfondo tiene que ver con la influencia ideológica del proceso hegemónico de medicalización capitalista de la muerte (Turner, 1987: 157-196), donde el sentido no puede venir de fuera de la institución y menos de la subjetividad de los otros en términos de consuelo, misericordia y compasión de los acompañantes, o sea una circularidad amorosa ${ }^{16}$ (Duch y Mélich, 2005) en la cual tiene que haber una recuperación de la confianza.

\footnotetext{
${ }^{15}$ Situación que pone de manifiesto el individualismo que priva en nuestro medio globalizado.

${ }^{16}$ No hay que perder de vista que creer qué es la muerte no es igual que saber qué es la muerte, por lo que este creer de los familiares pertenece a lo que Pascal señalaba como razones del "corazón", mismas que algunos también llaman intuitivas.
}

$\mathrm{Al}$ respecto, Rozenczweig (1998), en su trabajo sobre ética de la reanimación presenciada, advierte que los médicos excluyen a los familiares con base en un sentimiento de paternalismo, intentando protegerlos de la muerte, en la creencia de que es un evento traumático para ellos, y que traerá estrés al equipo que lo realiza; alegando que se puede violar el derecho de "confidencialidad" del paciente ${ }^{17}$, o temiendo que los familiares pudieran decidir prolongar inútilmente los esfuerzos, además de que se podrían evidenciar errores durante la reanimación. Una crítica más aguda la hace Berlinger (2002: 146-174) al hablar de la bioética cotidiana en el campo de la salud. Apunta este autor que "si no aceptamos los argumentos del bien común o del beneficio para los pacientes, la única forma de justificar una intervención médica es mediante el argumento de la inferioridad" del paciente, y las razones que se aluden para sostenerlo provienen de la religión, la cultura e incluso de la antropología de siglos pasados.

Por otra parte, la problemática que pudieran ofrecer los pacientes debe verse también desde una perspectiva psicosocial, ya que es sabido que en la relación médico-paciente los doctores experimentan entre 10 y $20 \%$ de dificultades en la interacción, pero las fuentes de dificultad estarán comprendidas parcialmente si no se analizan, en el comportamiento de los pacientes, aspectos tales como su estilo de acoplamiento con el equipo de salud. Por ejemplo, Maunder et al. (2006) documentaron que de los pacientes atendidos en Urgencias sólo 2\% presentó un estilo de acoplamiento seguro, $17 \%$ un estilo de preocupación, 19\% de restricción y 39\% de miedo. Esto amerita mayor investigación. Asimismo, Samples Twibell et al. (2008) señalan que la percepción de la RCPp se relaciona directamente con la forma en que el equipo de salud invita a los familiares a presenciarla.

Todo lo anterior nos habla de la necesidad de reconsiderar la dimensión ética de nuestras acciones como equipo de salud en relación con la RCPp en la sala de Urgencias. No tan sólo reflexionando sobre los principios éticos de justicia, autonomía, beneficencia (no maleficencia) y dig-

\footnotetext{
${ }^{17}$ Cuando la mayoría de las veces los familiares a los que se aleja fueron quienes acudieron a Urgencias con o por los pacientes, como la esposa(o), hija(o), padre o madre.
} 
nidad, sino también con base en "mi" responsabilidad de las acciones del otro, sobre todo en un contexto que no orienta sapiencialmente a la docta ignorancia del más allá de la muerte ${ }^{18}$.

\section{CONSIDERACIONES FINALES}

La tendencia actual de la globalización ${ }^{19}$ nos llevará sin duda a presenciar cada vez con más frecuencia situaciones como las planteadas en este trabajo, lo que nos debe conducir a prepararnos para encarar sabiamente estos retos. Por eso, los comentarios presentados en realidad son el inicio de una discusión más amplia que implica aspectos controversiales, como:

1) Los cuestionamientos acerca de la posible violación al derecho de confidencialidad cuando nuestros enfermos desean que sus familiares los acompañen.

2) El significado que para el equipo médico tiene la expresión "mi paciente"; hasta qué punto los pacientes son nuestros, y no como en realidad sucede, en el sentido de que el equipo de salud es corresponsable de un ser humano, quien detrás de sí tiene a su familia y su entorno sociocultural, y que deposita en profesionales su confianza porque le aqueja un problema que se interpreta como enfermedad.

3) Cuando el paciente desea que sus familiares lo acompañen y éstos deciden no hacerlo, ¿qué decisión debemos respetar?, ¿cómo podemos conciliar esta situación?

\footnotetext{
${ }^{18}$ Wittgenstein advierte que el sentido o la falta de sentido del "más allá" no son demostrables de modo alguno. Por eso, el consuelo ante un moribundo es el fundamento de estas relaciones humanas (Duch y Mélich, 2005)

${ }^{19}$ Se requiere contextualizar, profundizar y explorar de manera más extensa el concepto de RCPp tanto en la población derechohabiente como en el equipo de salud, donde se percibe una tensión que está dada por el proceso hegemónico de medicalización, cuyas principales características son: a) una concepción individualista del proceso salud-enfermedad-muerte; b) centralización de las intervenciones (racionalmente y legalmente justificadas) y de las decisiones por la urgencia en cuestión; c) mecanismos represivos desarrollados para el control, regulación y manejo de los cuerpos; d) el conservadurismo como justificación y resistencia al cambio; e) la contradicción derechos humanos-vigilancia social. Es decir, el control burocrático del hospital en aras de un concepto abstracto de salud del usuario.
}

4) Hasta qué punto las respuestas pueden orientarnos de manera no polarizada.

Indudablemente, bajo estos cuestionamientos se vuelve evidente el principio de beneficencia hacia el paciente y los familiares, al considerar que el paciente lo pide y los familiares piensan que su presencia efectivamente les puede ayudar en su proceso y su último trance. Ahora bien, ¿la presencia de familiares es benéfica para el equipo profesional que realiza la RCP, cuando la mayoría de nuestro personal no cree y no está listo para la RCPp (AHA, 2005b)? ¿Esta opinión de los médicos y las enfermeras es objetiva o tan sólo es una representación de temores personales o luchas de poder? Cuando Margunten et al. (2005) interrogaron a su equipo médico acerca de si quisieran que los familiares de los pacientes estuvieran presentes durante la RCPp, bajo el supuesto de que eran pacientes severamente enfermos o con un trauma importante, encontraron que hasta $86 \%$ de los participantes estaba a favor de la presencia de los familiares. Esta situación llevó a considerar una nueva pregunta: si bien nuestro deseo de "protección” frente a la muerte es aplicable a los enfermos y sus familiares, ¿también lo es a nuestra propia familia?

\section{Bibliografía}

American Heart Association (AHA), 2002, "Asistolia, algoritmo para corazón silencioso", en AVCA Manual para proveedores, Waverly Hispánica S. A, Brasil, pp. 117-118.

_ , 2005a, "Part 3, Overview of CPR", Circulation, vol. 112, Suppl. 1, pp. IV.12-IV.18

— 2005b, "Part 4, Adult Basic Life Support", Circulation, vol. 112, Suppl. 1, pp. IV.19-IV.29.

Berlinger, G., 2002, Bioética cotidiana, Siglo XXI, México.

Booth, M. G., L., Woolrich y J. Kinsella, 2004, "Family Witnessed Resuscitation in UK Emergency Departments: A Survey of Practice", European Journal of Anesthesiology, vol. 21, núm. 9, pp. 725-728.

Borgatti, S. P., 1992, Anthropac 4.0, Analityc Technologies, Columbia.

De Certau, Michael, 2000, La invención de lo cotidiano 1. Artes de hacer, Universidad Iberoamericana, México.

Duch, Lluís y Joan Carles Mélich, 2005, Escenarios de la corporeidad. La antropología de la vida cotidiana, Tretta, Madrid. 
Eichhorn, D. J., T. A. Meyers, C. E. Guzzetta, A. P. Clark, J. D. Klein, E. Taliaferro y A. O. Calvin, 2001, "Family Presence During Invasive Procedures and Resuscitation: Hearing the Voice of the Patient", American Journal of Nursing, vol. 101, núm. 5, pp. 48-55.

Elías, N., 1994, El proceso de la civilización. Investigaciones sociogenéticas y psicogenéticas, Fondo de Cultura Económica, México.

García de Alba García, J. E. y J. F. García Regalado, 2007, Opinión del personal médico y paramédico en las salas de Urgencias en los hospitales del centro del país sobre reanimación presenciada: una perspectiva mexicana de las recomendaciones 2000 de ACLS por la American Heart Association, ponencia, IV Congreso Internacional de Medicina de Urgencia, Puerto Vallarta.

García Regalado, J. F., 2007, Opinión de los pacientes y familiares que se encuentran hospitalizados en el área de Urgencias, adultos del HGR 46 sobre reanimación presenciada, tesis para obtener el título de la Especialidad de Urgencias, Universidad de Guadalajara, Instituto Mexicano del Seguro Social, Guadalajara.

Giddens, Antony, 1997, Modernidad e identidad del yo, Península, Barcelona.

Gómez Pérez, M. A. y J. A. Delgado Solís, 2000, Características de la población mexicana respecto a la muerte. Ritos y mitos de la muerte en México y otras culturas, Grupo Editorial Tomo, México.

Halm, M. A., 2005, "Family Presence During Resuscitation: A Critical Review of the Literature", American Journal of Critical Care, vol.14, núm. 6, pp. 494-511.

Huerta Torrijos, J., R. Díaz-Barriga Pardo y S. García Martínez, 2001, "Reanimación cardiopulmonar y cerebral. Historia y desarrollo", Revista de la Asociación de Medicina Crítica y Terapia Intensiva, vol. 15, núm. 2, pp. 51-60.

Lynch, I. y D. Medin, 2006, "Explanatory Models of the Illness, a Study within Culture Variation", Cognitive Psychology, vol. 53, núm. 4, pp. 285-309.

Lynn, J., J. Teno, R. S. Phillips, A. W. Wu, H. Desbiens, J. Harrold, M. T. Cleasens, N. Wenger, B. Kreinsling y S. Conrous, 1997, "Perceptions by Family Members of Dying Experience of Older and Seriously Ill Patients", Annals of Internal Medicine, vol. 126, núm. 2, pp. 97-106.

Mangurten, J. A., S. H. Scout, C. E. Guzzetta, J. S. Sperry, L. A. Vinson, B. A. Hicks, D. G. Watts y S. M. Scott, 2005, "Family Presence; Making Room”, American Journal of Nursing, vol. 105, núm. 5, pp. 40-48.

Martínez Ramírez, A., 2007, Gestión de la calidad en los servicios de salud, Universidad de Guadalajara, México.
Maunder, R. G., A. Panzer, M. Viljoen, J. Owen, S. Human y J. J. Hunter, 2006, "Physicians Difficulty with Emergency Department Patients is Related to Patients' Attachment Style", Social Science and Medicine, vol. 63, núm. 2, pp. 552-562.

McClenathan, B. M., K. G. Torrington y C. F. Uyehara, 2002, "Family Member Presence During Cardiopulmonary Resuscitation. A Survey of us and International Critical Care Professionals", Chest, vol. 122, núm. 6, pp. 2204-2211.

Meyers, T. A., D. J. Eichhhorn y C. E. Guzzetta, 1998, "Do Families Want to be Present During CPR", Journal of Emergency Nursing, vol. 24, núm. 6, pp. 400-405.

Meyers, T. A., D. J. Eichhorn, C. E. Guzzetta, A. P. Clark, J. D. Klein, E. Teliaferro y A. O. Calvin, 2000, “A Family Presence During Invasive Procedures and Resuscitation: The Experience of Family Members, Nurses and Physicians", American Journal of Nursing, vol. 100, núm. 2, pp. 32-42.

Moreland, P. y B. Manor, 2005, "Family Presence During Invasive Procedures and Resuscitation in the Emergency Department: A Review of the Literature", Journal of Emergency Nursing, vol. 31, núm. 1, pp. 58-72.

New Yorker, 2006, "Family Presence and Physician in Charge", 3 de abril, en línea: <http://www.nursingadvocacy.org/ news/2006/apr/03_new_yorker.htlm $>$, consultado el 17 de marzo de 2009.

Olvera Serrano, M. y O. Sabido Ramos, 2007, "Análisis sociológico de los miedos modernos”, Sociológica, vol. 22, núm. 64, pp. 119-149.

Robinson, S. M., S. Mackenzie Ross y L. Campbell Hewson, 1998, "Psychological effect of witnessed resuscitation on bereaved relatives”, Lancet, vol. 352, núm. 9128, pp. 614-617.

Rosenczweig, C., 1998, "Should Relatives Witness Resuscitation? Ethical Issues and Practical Considerations", Canadian Medical Association Journal, vol. 158, núm. 10, pp. 617-620.

Samples Twibell, R., D. Stela, Ch. Riwits, J. Wheatley, T. Riegle, D. Bousman, S. Cable, P. Caudill, S. Harrigan, R. Hollars, D. Johnson y A. Neal, 2008, "Nurses' Perceptions of Their SelfConfidence and Benefits and Risks of Family Presence During Resuscitation", American Journal of Critical Care, vol. 17, núm. 2, pp. 101-111.

Turner, B. S., 1987, Medical Power and Social Knowledge, Sage, Londres.

Warren, H., 1989, Diccionario de Psicología, Fondo de Cultura Económica, México.

Weller, Susan C. y A. Kimball Romney (eds.), 1988, Systematic Data Collection, Sage, Newbury Park, Londres, Nueva Delhi. 